\title{
On the liquid-glass transition line in monatomic Lennard-Jones fluids
}

\author{
M. Robles ${ }^{*}$ and M. López de Harof \\ Centro de Investigación en Energía \\ Universidad Nacional Autónoma de México \\ AP 34, Temixco, Mor. CP 62580, México. \\ (Dated: November 9, 2018)
}

\begin{abstract}
A thermodynamic approach to derive the liquid-glass transition line in the reduced temperature vs reduced density plane for a monatomic Lennard-Jones fluid is presented. The approach makes use of a recent reformulation of the classical perturbation theory of liquids [M. Robles and M. López de Haro, Phys. Chem. Chem. Phys. 3, 5528 (2001)] which is at grips with a rational function approximation for the Laplace transform of the radial distribution function of the hard-sphere fluid. The only input required is an equation of state for the hard-sphere system. Within the MansooriCanfield/Rasaiah-Stell variational perturbation theory, two choices for such an equation of state, leading to a glass transition for the hard-sphere fluid, are considered. Good agreement with the liquid-glass transition line derived from recent molecular dynamic simulations [Di Leonardo et al., Phys. Rev. Lett. 84, 6054(2000)] is obtained.

PACS numbers: 64.70.pf, 61.20.Gy, 05.70.Ce
\end{abstract}

The liquid-glass transition in Lennard-Jones (LJ) fluids has been a subject of interest for over twenty five years [1]. Recently, Di Leonardo et al. [2] using molecular dynamic simulations determined the liquid-glass transition line of a monatomic LJ system in the reduced density $\left(\rho^{*}\right)$ vs. reduced temperature $\left(T^{*}\right)$ plane. Here $\rho^{*}=\rho \sigma^{3}$ and $T^{*}=k_{B} T / \epsilon$, with $\rho$ the number density $k_{B}$ the Boltzmann constant, $T$ the temperature, and $\sigma$ and $\epsilon$ being the usual parameters of the LJ potential $\left(\phi_{L J}(r)=4 \epsilon\left(\sigma^{12} / r^{12}-\sigma^{6} / r^{6}\right)\right.$, where $r$ is the distance). Based on these results, they propose an off-equilibrium criterion to define the glass transition temperature $T_{g}$. This effective temperature compares rather well with the $T_{g}$ obtained from equilibrium calculations.

In a completely different context, a long time ago Hudson and Andersen [3] addressed the nature of the glass transition in monatomic liquids through an equilibrium calculation. In particular, in the case of the LJ fluid, they used the Weeks-Chandler-Andersen (WCA) perturbation theory of liquids [4] and two independent indications that a change in properties similar to a glass transition happens in the hard-sphere (HS) fluid at a packing fraction of $0.533 \pm 0.014$. Given the then scarce amount of simulation data available to compare with, their conclusion was that the use of the HS fluid as a reference fluid within the WCA scheme was adequate to derive the glass-transition line of monatomic fluids. As they already pointed out, for their approach to be useful in conection with glass formation in real systems, it is crucial that the HS fluid itself undergoes a glass transition. As it is well known, the existence of a glass transition in the HS fluid has been a debatable issue for a long time, but evidence from various sources seems to suggest that this is indeed the case [5, 6, 7, 8, 9, 10].

The main aim of this letter is to assess whether an equilibrium calculation, such as the one carried out by Hudson and Andersen [3], can still be useful to describe the new data on the liquid-glass transition line of LennardJones fluids. In a recent paper [11, we have reformulated the most popular schemes of the perturbation theory of liquids, namely the Barker-Henderson [12], the variational Mansoori-Canfiled/Rasaiah-Stell [13, 14] and the WCA 泊 schemes, using the HS fluid as the reference fluid. Our study focussed on the equilibrium properties (equation of state, critical point, liquid and solid branches of the reduced temperature vs. reduced density curve at coexistence and radial distribution function) of the Lennard-Jones fluid. All the calculations related to the different perturbative schemes that we reported in ref. 11] rely on a known analytical rational function approximation (RFA) to the radial distribution function (rdf) $g_{H S}(r)$ for the HS fluid developed by Yuste and Santos [15]. Their main idea is to propose that the Laplace transform $G(t)=\mathcal{L}\left[\operatorname{rg}_{H S}(r)\right]$, where $t$ is the Laplace transform variable, may be approximated by

$$
G(t)=\frac{t}{12 \eta} \frac{1}{1-e^{t} \Phi(t)},
$$

where $\eta=\pi / 6 \rho d^{3}$ is the packing fraction, $d$ being the hard-sphere diameter and $\Phi(t)$ is a rational function of the form

$$
\Phi(t)=\frac{1+S_{1} t+S_{2} t^{2}+S_{3} t^{3}+S_{4} t^{4}}{1+L_{1} t+L_{2} t^{2}} .
$$

The coefficients $S_{i}$ and $L_{i}$ are algebraic functions of $\eta$. They are determined by imposing two physical restrictions to the rdf [16], namely

1. The first integral moment of $h_{H S}(r)=g_{H S}(r)-1$ is well defined and non-zero.

2. The second integral moment of $h_{H S}(r)$ must guarantee the thermodynamic consistency of the compressibility factor $Z_{H S}=p /\left(\rho k_{B} T\right)(p$ being the 
pressure) and the isothermal susceptibility $\chi_{H S}=$ $\left(d\left(\eta Z_{H S}\right) / d \eta\right)^{-1}$.

These two conditions readily imply [15, 16] that

$$
\begin{aligned}
L_{1}= & \frac{1}{2} \frac{\eta+12 \eta L_{2}+2-24 \eta S_{4}}{2 \eta+1}, \\
S_{1}= & \frac{3}{2} \eta \frac{-1+4 L_{2}-8 S_{4}}{2 \eta+1} \\
S_{2}= & -\frac{1}{2} \frac{-\eta+8 \eta L_{2}+1-2 L_{2}-24 \eta S_{4}}{2 \eta+1}, \\
S_{3}= & \frac{1}{12} \frac{2 \eta-\eta^{2}+12 \eta L_{2}(\eta-1)-1-72 \eta^{2} S_{4}}{(2 \eta+1) \eta}, \\
L_{2}= & -3\left(Z_{H S}-1\right) S_{4}, \\
S_{4}= & \frac{1-\eta}{36 \eta\left(Z_{H S}-1 / 3\right)} \times \\
& {\left[1-\left[1+\frac{Z_{H S}-1 / 3}{Z_{H S}-Z_{P Y}}\left(\frac{\chi}{\chi_{P Y}}-1\right)\right]^{1 / 2}\right] }
\end{aligned}
$$

Here, $Z_{P Y}=\frac{1+2 \eta+3 \eta^{2}}{(1-\eta)^{2}}$ and $\chi_{P Y}=\frac{(1-\eta)^{4}}{(1+2 \eta)^{2}}$ denote the compressibility factor and isothermal susceptiblity arising in the Percus-Yevick theory. In order to close the approximation, a given equation of state for a HS fluid i.e. an explicit expression for the compressibility factor $Z_{H S}$ is needed.

All the perturbation schemes mentioned above introduce an effective (in general density and temperature dependent) diameter of the spheres as a fitting parameter to adjust some of the thermodynamic properties of the system of interest with respect to those of the reference HS system. Once this effective diameter is determined, one can infer by inversion the values of the temperature and density in the real system that correspond to a given packing fraction of the HS fluid. This fact was used in ref. 111 to determine the liquid and solid branches of the reduced temperature vs. reduced density curve at coexistence for a LJ fluid from the knowledge of the packing fractions for the fluid-solid transition $\eta_{F-S}=0.494$ and the solid-fluid transition $\eta_{S-F}=0.54$ in the HS fluid, respectively 17]. In a similar fashion, the liquid-glass transition line for the LJ system in the $\rho^{*}-T^{*}$ plane may be derived from the simple relationship

$$
\frac{\pi}{6} \rho^{*} d^{* 3}\left(\rho^{*}, T^{*}\right)=\eta_{g}
$$

where $\eta_{g}$ is the packing fraction corresponding to the glass transition in the HS fluid and we have introduced the reduced diameter (in units of $\sigma$ )denoted by $d^{*}$.

In order to proceed with such derivation, one has to specify the perturbation scheme and to know the value of $\eta_{g}$. As already stated above, Hudson and Andersen used the WCA and took $\eta_{g}=0.533 \pm 0.014$. In our case, we will consider two different equations of state for the HS fluid that allow us to calculate in a self-consistent way the value of $\eta_{g}$, and take the MC/RS scheme.

The first such equation of state is the Padé $(4,3)$ constructed from the knowledge of the first eight virial coefficients [18, 19]. The compressibility factor corresponding to this equation of state is given by

$$
Z_{43}=\frac{1+1.024385 \eta+1.104537 \eta^{2}-0.4611472 \eta^{3}-0.7430382 \eta^{4}}{1-2.985615 \eta+3.00700 \eta^{2}-1.097758 \eta^{3}}
$$

Note that, as was pointed out in previous work 8,20 , within the RFA the Padé(43) leads to a glass transition in the HS fluid at $\eta_{g}=0.5604$. It should also be borne in mind that this equation of state has a simple pole at a packing fraction very close to the fcc close-packing fraction [19].

On the other hand, the second equation of state is an ad-hoc approximation constructed in the following way. Since the well known Carnahan-Starling(CS) [21] equation of state has been shown to be very accurate throughout the complete fluid region and even in a small density range within the metastable regime but has a pole at $\eta=1$ which is clearly unphysical, we demand that the new compressiblity factor $Z_{\text {prop }}$ has a pole at the random close packing fraction $\eta_{r c p}$ (up to this point taken as a parameter) and that the first eight coefficients in its series expaexpansionnsion coincide with the same coefficients in the series expansion of the Carnahan-Starling compressibility factor. Thus, $Z_{\text {prop }}(\eta)$ is taken to be of the form

$$
Z_{\text {prop }}(\eta)=\frac{1+\sum_{i=1}^{5} a_{i} \eta^{i}}{\left(1-\eta / \eta_{r c p}\right)\left(1+b_{1} \eta+b_{2} \eta^{2}\right)}
$$

where the coefficients $a_{i}(i=1, \ldots, 5)$ and $b_{j}(j=1,2)$ depend on the value of $\eta_{r c p}$. In order to determine this value, we further assume that, according to the criterion that has been used earlier [8, 20], a glass transition in the HS fluid (considered to be a second order phase transition) occurs at $\eta_{g}$ (also unspecified at this stage) if $S 4$ vanishes for $\eta_{g}$. The above conditions lead to the 
following set of equations:

$$
\begin{aligned}
S_{4}\left(\eta_{g}\right) & =0, \\
Z_{\text {glass }}\left(\eta_{g}\right) & \equiv \frac{A}{1-\eta_{g} / \eta_{r c p}} \\
& =Z_{\text {prop }}\left(\eta_{g}\right), \\
\chi_{\text {glass }}\left(\eta_{g}\right) & \left.\equiv\left(\frac{\partial\left(\eta Z_{\text {glass }}\right)}{\partial \eta}\right)^{-1}\right|_{\eta=\eta_{g}} \\
& =\frac{\chi_{P Y}\left(\eta_{g}\right)\left(Z_{P Y}\left(\eta_{g}\right)-1 / 3\right)}{\left(Z_{\text {prop }}\left(\eta_{g}\right)-1 / 3\right)},
\end{aligned}
$$

where $A$ is a constant and in writing the compressibility factor for the glass we have taken the form proposed by Speedy [5, 7]. The set of equations (12)-(14) together with (11) allow us to determine $\eta_{r c p}, A$ and $\eta_{g}$ in a selfconsistent way. The results are $\eta_{r c p}=0.6504, A=2.780$ and $\eta_{g}=0.5684$, which are well within the range of published values for these quantities. In turn the value of $\eta_{r c p}$ leads to the explicit form of $Z_{\text {prop }}(\eta)$, namely

$$
Z_{\text {prop }}(\eta)=\frac{1+0.153555 \eta-0.428376 \eta^{2}-2.7987 \eta^{3}-0.317417 \eta^{4}-0.105806 \eta^{5}}{1-3.84644 \eta+4.9574 \eta^{2}-2.16386 \eta^{3}}
$$

In the range $0 \leq \eta \leq 1$ this equation of state only presents a simple pole at $\eta=\eta_{r c p}$.

In order to illustrate the numerical accuracy of these two equations of state in the metastable region, in Fig. 11 we display the inverse of the contact values of the rdf $\left(g\left(d^{+}\right)^{-1}=4 \eta /\left(Z_{H S}-1\right)\right)$ derived from them as a function of the packing fraction and compare the results with the simulation data obtained by Rintoul and Torquato [22]. In this figure we have also included the predictions of the corresponding equations of state for the glass adopting the form suggested by Speedy [0, 7].

For the sake of making the paper self contained, we now recall the condition required to derive the temperature and density dependent diameter within the MC/RS perturbation scheme (for details see refs. 111, 13, 14). Here the effective diameter is chosen to minimize the

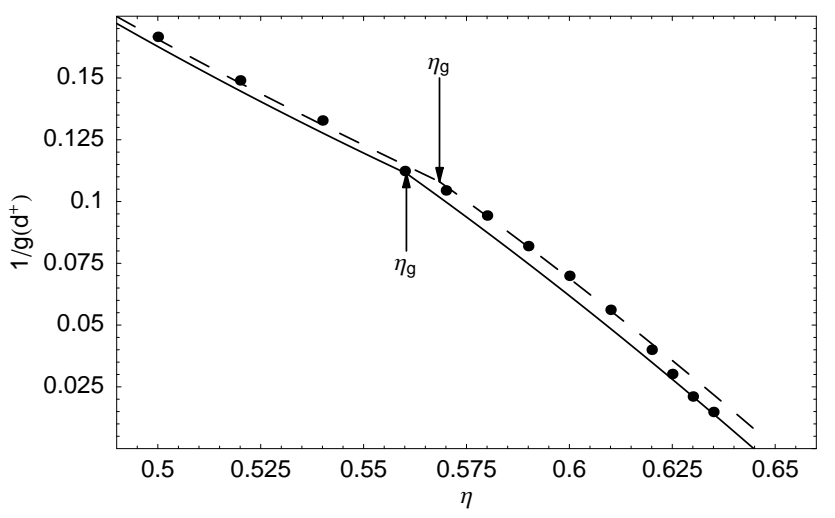

FIG. 1: Inverse of the contact values of the rdf as derived from $Z_{43}$ (continuous line) and $Z_{\text {prop }}$ (dashed line) as a function of $\eta$. The region corresponding to the glass has been obtained from $Z_{\text {qlass }}$ and in the case of $Z_{43}, A=2.765$ and $\eta_{\text {rcp }}=$ 0.6448 20]. The full symbols are simulation results of ref. [22] and the arrows indicate the location of $\eta_{g}$ for each case.
Helmholtz free energy of the perturbed system. To first order in $\beta=1 / T^{*}$ this diameter is determined from the equation 11

$$
\begin{aligned}
\frac{\partial}{\partial d^{*}}\left[\int_{0}^{\rho^{*}} \frac{Z_{H S}-1}{\rho} d \rho+4\left(\frac{1}{d^{*}}\right)^{6} \beta \times\right. \\
\left.\quad \int_{0}^{\infty} \frac{t}{1-e^{t} \Phi(t)}\left(\left(\frac{1}{d^{*}}\right)^{6} \frac{t^{10}}{10 !}-\frac{t^{4}}{4 !}\right) d t\right]=0 .
\end{aligned}
$$

Clearly in solving this equation it should be understood that $Z_{H S}$ and $\Phi(t)$ have been expressed as functions of $\rho^{*}$ and $d^{*}$.

Using the effective diameters determined from eq. (16) with either $Z_{43}$ or $Z_{\text {prop }}$ and the condition given by eq. (9) (with the $\eta_{g}$ value corresponding to each equation of state) we have determined the liquid-glass transition lines in the $\rho^{*}-T^{*}$ plane for the LJ fluid. These are shown in Fig. 2 where we have also included the recent simulation results of Di Leonardo et al. [2]. As clearly seen in the figure, not only the qualitative trend observed in the simulations is reproduced, but also the quantitative agreement is rather good, specially for the case of $Z_{\text {prop }}$. A remarkable aspect of these results is that they have been derived self-consistently with no free parameters.

One question that immediately arises is to what extent the above results depend on the MC/RS perturbation scheme and the choices for $Z_{H S}$. Concerning the first issue, which is particularly relevant in view of the fact that the previous calculation by Hudson and Andersen [3] used the WCA, we have checked that the performance of this latter scheme is much poorer. This may reflect that the choice of minimizing the Helmholtz free energy of the perturbed system to derive the effective diameter may be more astringent than equating the isothermal compressiblities of the actual and the HS reference system. Something similar applies to the Barker-Henderson 


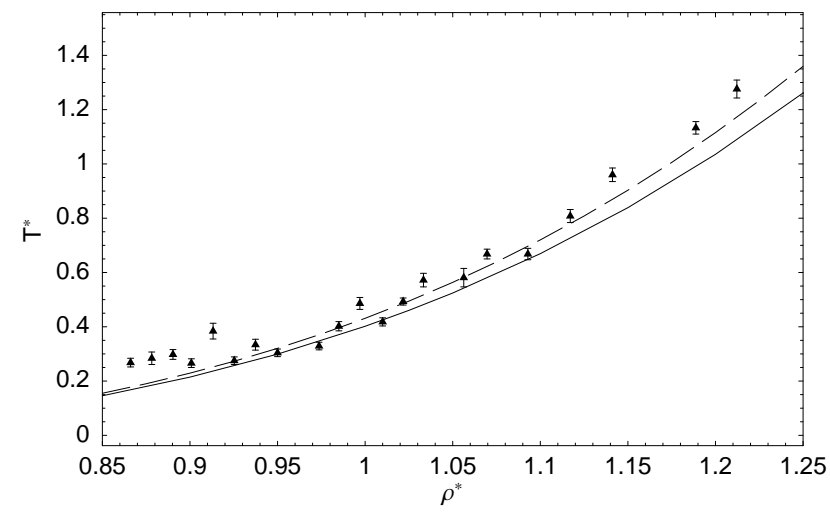

FIG. 2: Liquid-glass transition lines as obtained with the $\mathrm{MC} / \mathrm{RS}$ perturbation scheme using $Z_{43}$ (continuous line) and $Z_{\text {prop }}($ dashed line). The points are the simulation data of Di Leonardo et al. in ref [2]

perturbation theory in which the lack of density dependence of the effective diameter does not reproduce the desired trend. As far as the second issue is concerned, we have also performed calculations taking the CS equation of state (which does not lead to a glass transition in the RFA formulation) and adjusted the value of the packing fraction $\eta_{g}$ until good agreement with the simulation data of Di Leonardo et al. 22] was obtained. In fact for $\eta_{g}=0.55$ we get results pretty close to those derived using $Z_{\text {prop }}$. The above suggests that if the exact equation of state of the HS system (including a glass transition) were available then the use of perturbation theory and the RFA approach would yield a very accurate description of the liquid-glass transition line of the LJ fluid.

In conclusion, in this paper we have shown that an equilibrium approach to the glass transition in LJ fluids, much in the same spirit as discussed by Baeyens and Verschelde [6] in the case of HS fluids, is wholly compatible with the molecular dynamics simulation results for the liquid-glass transition line reported recently [2]. Perhaps the key aspect of our approach is its self-consistency and the provision of a theory with no free parameters.
Furthermore, the application of the same approach for other monatomic fluids may be laborious, but in principle should follow the same steps.

* Electronic address: mrp@cie.unam.mx

$\dagger$ Electronic address: malopez@servidor.unam.mx

[1] See for instance, A. Rahman, M. Mandell, and J. McTague, J. Chem. Phys. 64, 1564 (1976).

[2] R. Di Leonardo, L. Angelani, G. Parisi, and G. Ruocco, Phys. Rev. Lett. 84, 6054 (2000).

[3] S. Hudson and H. C. Anderssen, J. Chem. Phys. 69, 2323 (1978).

[4] J. D. Weeks, D. Chandler, and H. C. Andersen, J. Chem. Phys. 53, 149 (1971).

[5] R. J. Speedy, J. Chem. Phys. 100, 6684 (1994).

[6] B. Baeyens and H. Verschelde, Z. Phys. B 102, 255 (1997).

[7] R. J. Speedy, Mol. Phys. 95, 169 (1998).

[8] M. Robles, M. López de Haro, A. Santos, and S. Bravo Yuste, J. Chem. Phys. 108, 1290 (1998).

[9] W. van Megen and S. M. Underwood, Nature 362, 616 (1993).

[10] W. van Megen, T. C. Mortensen, S. R. Williams, and J. Müller, Phys. Rev. E. 58, 6073 (1998).

[11] M. Robles and M. López de Haro, Phys. Chem. Chem. Phys. 3, 5528 (2001).

[12] J. A. Barker and D. Henderson, J. Chem. Phys. 47, 2856 (1967).

[13] G. A. Mansoori and F. B. Canfield, J. Chem. Phys. 51, 4958 (1969).

[14] J. Rasaiah and G. Stell, Mol. Phys. 18, 249 (1970).

[15] S. B. Yuste and A. Santos, Phys. Rev. A 43, 5418 (1991).

[16] M. Robles and M. López de Haro, J. Chem. Phys. 12, 4648 (1997).

[17] J. P. Hansen and L. Verlet, Phys. Rev. 184, 151 (1969).

[18] E. J. J. van Rensburg, J. Phys. A 26, 4805 (1993).

[19] I. C. Sanchez, J. Chem. Phys. 10, 7003 (1994).

[20] S. Bravo Yuste, M. López de Haro, and A. Santos, Phys. Rev. E 53, 4820 (1996).

[21] N. F. Carnahan and K. E. Starling, J. Chem. Phys. 51, 635 (1969).

[22] M. D. Rintoul and S. Torquato, J. Chem. Phys. 105, 9258 (1997). 